\title{
Computed Tomography Image Enhancement Using Cuckoo Search: A Log Transform Based Approach
}

\author{
Amira S. Ashour ${ }^{1}$, Sourav Samanta ${ }^{2}$, Nilanjan Dey ${ }^{3}$, Noreen Kausar ${ }^{4}$, \\ Wahiba Ben Abdessalemkaraa ${ }^{5,6}$, Aboul Ella Hassanien ${ }^{7}$ \\ ${ }^{1}$ Department of Electronics \& Electrical Communications Engineering, Faculty of Engineering, Tanta University, \\ Tanta, Egypt \\ ${ }^{2}$ Department of Computer Science \& Engineering, University Institute of Technology, \\ Burdwan, India \\ ${ }^{3}$ Department of Computer Science \& Engineering, Bengal College of Engineering Technology, Durgapur, \\ India \\ ${ }^{4}$ Faculty for Mathematics and Computer Science, Alfaisal University, Riyadh, Saudi Arabia \\ ${ }^{5}$ College of Computers and Information Technology, Taif University, Taif, Saudi Arabia \\ ${ }^{6}$ RIADI-GDL Laboratory, The National School of Computer Sciences (ENSI), Manouba, Tunisia \\ ${ }^{7}$ Scientific Research Group, Cairo University, Cairo, Egypt \\ Email:amirasashour@yahoo.com, sourav.uit@gmail.com, neelanjan.dey@gmail.com, \\ noreenkausar88@yahoo.com, wahiba.bak@gmail.com, aboitcairo@gmail.com
}

Received 25 July 2015; accepted 28 August 2015; published 31 August 2015

Copyright (C) 2015 by authors and Scientific Research Publishing Inc.

This work is licensed under the Creative Commons Attribution International License (CC BY).

http://creativecommons.org/licenses/by/4.0/

(c) (i) Open Access

\section{Abstract}

Medical image enhancement is an essential process for superior disease diagnosis as well as for detection of pathological lesion accurately. Computed Tomography (CT) is considered a vital medical imaging modality to evaluate numerous diseases such as tumors and vascular lesions. However, speckle noise corrupts the CT images and makes the clinical data analysis ambiguous. Therefore, for accurate diagnosis, medical image enhancement is a must for noise removal and sharp/clear images. In this work, a medical image enhancement algorithm has been proposed using log transform in an optimization framework. In order to achieve optimization, a well-known meta-heuristic algorithm, namely: Cuckoo search (CS) algorithm is used to determine the optimal parameter settings for log transform. The performance of the proposed technique is studied on a low contrast CT image dataset. Besides this, the results clearly show that the CS based approach has superior convergence and fitness values compared to PSO as the CS converge faster that proves the efficacy of the CS based technique. Finally, Image Quality Analysis (IQA) justifies the robustness of the proposed enhancement technique. 


\section{Keywords}

\section{Meta-Heuristic, Cuckoo Search, Image Enhancement, Medical Imaging, Log Transform}

\section{Introduction}

Image enhancement is one of the protruding research areas in image processing as it is more favorable in numerous applications, namely: military [1], satellite image processing [2], geographical imaging [3] and medical image processing [4]. Its techniques are used to process a given image to recognize its features easily [5] clarifying its details that cannot be immediately observed in the original image, especially when the image is corrupted with high level of speckle noise [6] or has insufficient contrast [7] [8]. Since medical images are often deteriorated by noise due to different sources of interference and other equipments' artifact that affects the measurement processes in imaging and data acquisition systems. Thus, advanced medical equipments have attracted much attention to improve medical image technologies as in [9]-[12]. Since the desired region of interest (ROI) is not well distinguishable from the background, the role of image enhancement augments is to improve the visibility of significant features in a medical image to facilitate the diagnosis process and remove/reduce noise.

Basically, image enhancement is the process of transforming/mapping digital images so that the results are more fitting for further image analysis [13]. According to the objective of image enhancement methods, they are broadly categorized into: spatial domain and frequency domain enhancement [14] [15]. Commonly, there are four categories of enhancement techniques based on the kind of transformation employed, namely: point operations (e.g. histogram equalization), spatial operation (e.g. median filtering), transform operations (e.g. homomorphic filtering) and pseudocoloring [16].

Typically, spatial domain techniques directly deal with the image pixels that are controlled to attain the required enhancement. These techniques such as the logarithmic transforms, histogram equalization and power law transforms. They are mainly useful for directly modifying the individual pixel gray level values and thus the entire image overall contrast [17]. Log transformation is one of the basic well-known image enhancement techniques of the spatial domain that can be efficiently used for dark image contrast enhancements. It maps a narrow range of low input gray level values in a broad range of output values [18] [19]. As log functions are most valuable when the input gray level values have an exceptionally large range of values. During log transformation, the image dark pixels are expanded as compare to the higher pixel values that are type of compressed in log transformation that leads to image enhancement.

Trial and error and low level mathematical operators [20] are widely used in medical studies. However, medical image processing problems are empirical and/or heuristic in nature. Therefore, the experience-based heuristic method is designed to provide a solution which that may not be optimal, but can efficiently achieve a certain set of goals. Since image enhancement algorithm optimization parameters for every image type is an exhaustive task, image enhancement becomes a complex optimization problem leading to the use of a new class of algorithms; Meta-heuristics techniques that can provide superior results in a reasonable amount of time [21].

A meta-heuristic technique is a set of algorithms used to define heuristic methods for various problems. These techniques involve defining an objective evaluation criterion to evaluate the fitness of the enhanced image under consideration. Moreover, the algorithm must be able to optimize the parameters of the transformation function, based on the input image. Thus, such techniques have been proposed for automatic image enhancement [22]. Genetic Algorithms (GAs) are considered adaptive heuristic search algorithms based on the evolutionary ideas of natural selection and genetics. GA is valuable in image enhancement as it is a derivative-free and stochastic optimization method [23]. In addition, all meta-heuristic algorithms use a certain randomization and local search and tend to be suitable for global optimization [24]. Several meta-heuristics have been proposed to deal with the computationally inflexible problems such as genetic algorithm and simulated annealing [25], Particle swarm optimization (PSO) [26] [27], Cuckoo search [28] [29], Honey bee (HB) [30]. Among all the meta-heuristic algorithms, the Cuckoo Search (CS) algorithm is very proficient and less time consuming [31]. The CS has the advantage of both local search capabilities and guaranteed global convergence for automatic medical image enhancement application.

There are various equipments for medical imaging (X-Ray, CT, MRI, etc.) that encompasses information 
about heart, brain, nerves and other body organs providing physicians with a precise analysis of diagnosis and diseases. In the case of CT, numerous mathematical and medical applications can be applied to determine whether the normal tissue has been infected by the mutations of the cancer cell [32]. The computerized axial tomography (CAT), known as CT, proves its efficiency in medical image processing. It is considered one of the most important events in the application of image processing in medical diagnosis. Medical image enhancement focuses on solving the poor contrast and higher level noise of the medical image. The key step for medical image enhancement is based on selecting the suitable technique to: clarify the images for better human vision via removing noise/blur effect, to increase the contrast and to highlight the important details. In CT, pre-processing and enhancement techniques are used to improve the detection of the region of interest from CT images. Due to new medical instruments used in the medical field, much consideration is directed to medical image enhancement technologies. Since the CT suffers from blurred image and metal artifacts, the CT image enhancement becomes a must.

In [33], an optimal enhancement was proposed using PSO for image enhancement, combining both log transform and PSO. The experimental results proved the PSO superiority compared to histogram equalization, linear contrast stretching and genetic algorithm based image enhancement. Consequently, the low contrast CT image of the liver is used to test the proposed algorithm where CS optimizes the parameters of the log transformation to enhance the images. The obtained result is also compared to the PSO based enhancement technique to evaluate the performance of the proposed study, as CS is a well established meta-heuristic algorithm.

Therefore, in this study a medical image enhancement algorithm has been proposed using log transform based Cuckoo search (CS) algorithm that optimize its paprameters. A low contrast CT image data set is used to test the proposed method performance. In addition, the Image Quality Analysis (IQA) is used to justify the robustness of the proposed enhancement technique.

The rest of the paper is constructed as follows: Section 2 demonstrates related work on medical image enhancement. Then, Section 3 includes the material and methods used, including the log transform algorithm and the Cuckoo search algorithm. The proposed method of Cuckoo Search optimization algorithm with log transform for CT image enhancement is included in Section 4. Section 5 includes results and discussion. Finally, the conclusion and future work in Section 6.

\section{Related Works on Medical Image Enhancement}

In 1960, digital image processing techniques began; consecutively medical imaging started in 1970s [34]. CT is a non-invasive medical examination where algorithms are used to construct an image representing a "slice" through the object [35]. Recent related works to CT de-noising, enhancement and classification can be addressed as follows. Recent wavelet thresholding based de-noising methods have proven its capability with high-frequency signal details [36]. The threshold at certain scale is constant for all wavelet coefficients in the standard wavelet thresholding based noise reduction methods. A dual-tree complex wavelet based image de-noising method was introduced in [37]. In [38] a technique for image de-noising in the filtered domain based on the characteristics of the Empirical Mode Decomposition (EMD) and the wavelet technique were presented. An efficient noise reduction technique for CT images using windows-based multi-wavelet transformation and thresholding is proposed in [39]. In the year 2007 [18], an enhancement technique based on logarithmic transforms coefficient adaptive histogram equalization (LTAHE) has been introduced. The method was based on the properties of logarithmic transform domain histogram as well as the contrast limited adaptive histogram equalization. The performance of the algorithm was compared quantitatively to the classical histogram equalization. The experimental results showed that the proposed algorithm performance was alongside classical histogram equalization.

In [40], an image enhancement technique using CS algorithms and morphological operations was presented. The contrast value of the image was attained by calculating the fitness through the CS algorithm. In order to improve the quality of the image; the best contrast value of the image was selected and morphological operations were done by adjusting the intensity parameters. In [41], a CS based algorithm for low quality fingerprint image contrast enhancement was proposed. The authors used a new objective function as a quality metric for global fingerprint image enhancement along with the CS for the gray level mapping technique for contrast enhancement. The results showed that the suggested CS algorithm can enhance the fingerprint images, on the general level of both noise eradication and quality metrics. In the same year, Bhandaria et al. [42], introduced a novel 
contrast enhancement approach based on the CS algorithm and discrete wavelet transform-singular value decomposition (DWT-SVD) for quality improvement of the low contrast satellite images. The CS algorithm has been used to optimize each sub-band of the DWT and then acquired the singular value matrix of the low threshold sub-band image and ultimately used the inverse DWT to reconstruct the enhanced image. The results proved the superiority of the proposed method performance in terms of the Peak signal to noise ratio, Mean and Standard Deviation over the conventional techniques. In [43], the Cuckoo Search algorithm has been used to tune the image enhancement functions for peak performance. For assessment a comparison to both the Genetic Algorithms and the Particle Swarm Optimization, Histogram Equalization and Linear Contrast Stretch techniques has been conducted. The experimental results have demonstrated the capability of the CS algorithm in optimizing the enhancement functions. Currently, by the year 2015 in [44], the authors have been presented an image enhancement approach using a combination of the CS algorithm with Morphological Operation. The best contrast value of an image has been selected using the CS algorithm, then the morphological operations have to be performed. The results have been demonstrated that the proposed approach was converted into original color image without noise and adaptive process in order to enhance the quality of images.

\section{Materials and Methods}

\subsection{Function Overview}

Commonly, image enhancement algorithms depend on the objective to be achieved as well as the application. The visual evaluation of image quality is highly subjective. Therefore, an urgent need for any image enhancement approach arises.

\subsubsection{Transformation Function}

Generally, any image can be defined as a two-dimensional function, $R(x, y)$ where $(x, y)$ are spatial coordinates, and the value of $R$ at any pair of coordinates at locations $(x, y)$ is called the intensity or gray-level (tone) of the image at that point. Classical image enhancement methods aim to improve the image contrast and sharpness of edges and to remove the noise as well. In order to solve this problem, a Logarithmic image processing (LIP) model was originally developed by Jourlin and Pinoli [45]. The LIP is a mathematical framework which provides a set of generalized adaptation of subtraction, addition, multiplication, convolution, etc. for signal and image processing. The brightness of an image are considered in the LIP model as the intensity of light that passes through a light filter with absorption function $R(x, y)$. The absorption function is defined as the percentage of the incident light being absorbed by the light filter, also named as gray tone function. Therefore, an image can be represented by the absorption function. A realistic example of the light filter is the CT images, $\mathrm{X}$-ray film, etc.

Consequently, instead of representing the CT image by its pixel brightness, it can be represented using the gray tone function as the image enhancement can be done through the gray level transformations. Assume an original image that has $M$ columns and $N$ rows. The enhancement occurs at each pixel at location $M \times N$ of the original image. The enhancement can be illustrated in [46],

$$
P(x, y)=T[R(x, y)]
$$

where, the transformation function $T$ has a gray value of the input image pixels $(x, y)$ denoted by $R(x, y)$ and $P(x, y)$ is the enhanced image gray value of the pixels $(x, y)$.

For the local information extraction, a window of $n \times n$ size is used. The transformation is defined as follows:

$$
P(x, y)=B(x, y)[R(x, y)-c \times z(x, y)]+z(x, y)^{a}
$$

As $a$ and $c$ are parameters. Over $n \times n$ window, the local mean of the pixel $(x, y)^{\text {th }}$ of the input image is;

$$
z(x, y)=\frac{1}{n \times n} \sum_{i=0}^{n-1} \sum_{j=0}^{n-1} R(i, j)
$$

The enhancement function $B(x, y)$ that holds both the local and global information is: 


$$
B(x, y)=\frac{k \cdot A}{\sigma(x, y)+b}
$$

where, $k$ and $b$ are two parameters. The global mean is defined as;

$$
A=\frac{1}{M \times N} \sum_{x=0}^{M-1} \sum_{y=0}^{N-1} R(x, y)
$$

In addition, the local standard deviation of the input image pixel $(x, y)^{\text {th }}$ for the $n \times n$ window is given as;

$$
\sigma(x, y)=\sqrt{\frac{1}{n \times n} \sum_{i=0}^{n-1} \sum_{j=0}^{n-1}(R(i, j)-z(x, y))^{2}}
$$

By substituting from (4) into (2), the new transformation function is obtained where the contrast of the image is stretched,

$$
P(x, y)=\frac{k \cdot A}{\sigma(x, y)+b}[R(x, y)-c \times z(x, y)]+z(x, y)^{a}
$$

The center of the stretch is $z(x, y)$. The four parameters mentioned before are to produce large variations in the input image. In order to enhance the image, Lee's algorithm with the log transform is used to increase the overall contrast and sharpness.

\subsubsection{Logarithmic Image Processing (Log Transform)}

The gray tone (intensity) function is restricted as the brightness of an image is usually restricted to an interval; $L=$ $[0,255]$ for an 8-bit image. Where, the value "zero" means no absorption, while the value $L$ corresponds to a totally opaque image. In [47], a modified method for the implementation of Lee's algorithm facilitates the good quality enhancement. For an 8-bit image, $L=256$, the image intensity function $F$ is transformed to the gray tone function as,

$$
R: F(x, y)=L-R(x, y)
$$

Subsequently, the gray tone function is transformed to the normalized negative gray tone function through the following equation,

$$
\bar{f}=1-\frac{f}{L}
$$

Afterward, the logarithm of (9) is applied using Lee's algorithm as follows,

$$
\log \left(\bar{f}^{\prime}(x, y)\right)=\beta \log (\bar{k}(x, y))+\psi[\log (\bar{f}(x, y))-\log (\bar{k}(x, y))]
$$

where, $\bar{k}(x, y)$ is the mean value of $\bar{f}$ in a window of size $(\xi \times \xi)$ centered on $(x, y)$. Through converting $f^{\prime}$ back to the original scale, the enhanced output image $F^{\prime}$ is obtained.

The three control parameters for the algorithm according to (10) are;

1) $\beta$ is used to adapt the image dynamic range. The smaller the value of $\beta$, the brighter the image will appear.

2) $\psi$, is used to control the image sharpness. For sharper image the value of $\psi$ is chosen to be large within its range $\psi \in[0,255]$.

3) $\xi$, is used to control the window size.

An initial range of $[0,2.0]$ is used for both $\beta$ and $\psi$, while the window size $\xi$ is chosen to be 3 for the proposed CT image enhancement application through experiment. Then, a selection of the optimal value of $\beta$ and $\psi$ to enhance the dark CT image is performed based on the Cuckoo search algorithm to obtain the optimal parameters' values of the log transformation. This leads to an enhanced CT image.

\subsection{Evaluation Criteria}

In order to evaluate/measure the quality of the enhanced image, an objective function is required that has three 
performance measures are:

- Edge intensities sum.

- Number of edges, as higher intensity of edges and more number of them as compared to the original image indicate good enhanced image.

- Entropy value, which contains information about the image.

The objective function mentioned is:

$$
F\left(I_{e}\right)=\log \left(\log \left(E\left(I_{s}\right)\right)\right) \times \frac{n_{\text {edges }}\left(I_{s}\right)}{M \times N} \times H\left(I_{e}\right)
$$

$I_{e}$ is the enhanced image created using the transformation function in (2). While, $I_{s}$ is the edge image using Sobel edge detection, which is one of the efficient automatic threshold detector algorithms. $E\left(I_{s}\right)$ is the sum of $M \times N$ pixel intensities of Sobel edge image $I_{s}$ and $n_{\text {edges }}$ is the number of pixels, whose intensity value is above a threshold in $I_{s}$. Using the enhanced image $I_{e}$ to produce an edge image as follows,

$$
I_{s}(i, j)=\sqrt{\delta m I_{e}(i, j)^{2}+\delta n I_{e}(i, j)^{2}}
$$

where,

$$
\begin{gathered}
\delta \cap I_{e}(i, j)=P I_{e}(i-1, j+1)+2 P I_{e}(i, j+1)+P I_{e}(i+1, j+1) \\
-P I_{e}(i-1, j-1)-2 P I_{e}(i, j-1)-P I_{e}(i+1, j-1) \\
\delta \cap I_{e}(i, j)=P I_{e}(i-1, j+1)+2 P I_{e}(i, j+1)+P I_{e}(i+1, j+1)-P I_{e}(i-1, j-1) \\
-2 P I_{e}(i, j-1)-P I_{e}(i+1, j-1)
\end{gathered}
$$

The entropy value is calculated using the histogram on the enhanced image $I_{e}$, as:

$$
H\left(I_{e}\right)=\sum_{i=0}^{255} h_{i} \log _{2}\left(h_{i}\right)
$$

where, the histogram transformation is considered one of the fundamental processes for contrast enhancement of gray level images. As, $h_{i}$ is the probability of occurrence of the $i^{\text {th }}$ intensity value of $I_{e}$ image.

\subsection{Cuckoo Search based Image Enhancement}

Approximately, all meta-heuristics use some form of stochastic components and try to reproduce the best features in nature, especially biological systems. The selection of fitness and adaptation to the environment are essential characteristics that the meta-heuristic algorithms attained. Traditionally, optimization techniques can be divided into two types [48]:

- Direct method: is based on the objective function and constraints. This method is slow and requires numerous function evaluations for convergence.

- Gradient-based method: used the $1^{\text {st }}$ or $2^{\text {nd }}$ order derivative of the objective function (OF) and/or constraints for guiding the search process. This method converges to an optimal solution; having less efficiency in non-differentiable or discontinuous problems. Also, it is difficult and unstable because the objective functions have a number of peaks.

Due to these drawbacks, the researchers develop the meta-heuristic algorithms to resolve the engineering optimization problems. Meta-heuristic algorithms have many methods, such as: simulated annealing (SA), Genetic algorithm (GA), Cuckoo Search (CS), particle swarm optimization (PSO), Firefly algorithm (FA), etc. The CS algorithm has obtained great interests and is found to be proficient as in [49]. Hence, in the current paper the Cuckoo Search algorithm via Lévy flights is used to optimize the parameters of the log transform enhancement method. The CS algorithm is based on the considerable breeding behaviour such as brood parasitism of convincing species of Cuckoos. The CS algorithm is considered a new meta-heuristic algorithm for solving the optimization problems. It is motivated by the obligate brood parasitism of some Cuckoo species laying their eggs in the other host birds' nest. Several host birds can employ direct conflict with the intruding Cuckoos. The algorithm merged with another key feature which is the Lévy flight behaviour of fruit flies and some of the birds. The Lévy flights are considered a class of random walks primarily devised. In the CS algorithm, each egg 
represents a new better solution to replace weak solutions in the nest. Using the simplest structure, each nest has one egg.

In [50], three idealized rules that describe the Cuckoo Search algorithm are:

- At a time, each cuckoo lays one egg and leaves it in a randomly chosen nest;

- In the next generations, the best nests with high quality of eggs (solutions) will be carried over;

- The number of existing host nests is fixed and a host can discover an alien egg with a probability $P_{a} \in[0,1]$. In this case, the host bird can either throw the egg away or abandon the nest so as to build a completely new nest in a new location.

For simplicity, this last assumption can be estimated by a fraction $P_{a}$ of the $w$ nests being replaced by new nests (with new random solutions at new locations).The solution fitness can be proportional to the objective function for a maximization problem. Based on these three rules, the basic steps of the Cuckoo Search (CS) are summarized in the next section. In this algorithm, when generating a new solution, a balanced arrangement of both local and global explorative random walk is controlled by a switching parameter $P_{a}$. The following equation represents the local random walk:

$$
x_{i}^{t+1}=x_{i}^{t}+\alpha s \otimes H\left(P_{a}-\varepsilon\right) \otimes\left(x_{j}^{t}-x_{k}^{t}\right)
$$

where, $x_{i}^{t}$ and $x_{k}^{t}$ are two different solutions chosen randomly by indiscriminate permutation, $H(u)$ is a Heaviside function, $\varepsilon$ is a random number drawn from a uniform distribution and $s$ is the step size.

The global random walk performed using Lévy flight is given by;

$$
x_{i}^{t+1}=x_{i}^{t}+\alpha Q(s, \lambda)
$$

Here, $\alpha>0$ is the step size, $\left(s \gg s_{0}>0\right)$ and $Q(s, \lambda)$ is the characteristic scale of the problem of interest.

\section{Proposed Method Using Cuckoo Search Optimization Algorithm for Log Transform Parameters Optimization for CT Images}

The CS proves its efficiency for better convergence towards global optimization than any other global search algorithm. Therefore, it is used through this work to support the log transformation. The parameter optimization in the log transformation-based image enhancement technique using CS algorithm is introduced in this work. According to (10), $\beta$ and $\psi$ are the two parameters that have to be optimized in the log transform and the value of the third parameter (window size control $\xi$ ) will be set to 3 .

In addition, in the CS Algorithm, each nest indicates the candidate solution that basically set to be 25 nests. Conversely, in the problem under concern, the search space is limited which leads to very quickly premature convergence towards the optimal solution with 25 nests. Thus, less number of nests are preferred and passed on trial and error, it is obtained that using 10 nests is suitable for our current experimental study.

In the proposed method, the following assumptions and steps will be tracked:

1) Each Cuckoo nest is represented by $\beta$ and $\psi$. Clearly, each one belongs to the two dimensional space ( $d=2$ where, $d$ defines the dimensions of the problem).

2) The range of the parameters $\beta$ and $\psi$ has taken within the interval [0 to 2.0].

3) The number of nests is 10.

4) Each nest is used to enhance the original image using (10).

5) Fitness of each nest is determined.

6) The new nest is generated according to the best nest of the previous iteration.

7) When the algorithm reaches its maximum iteration, it stops and gives the global optimum solution containing values of $\beta_{g}$ and $\psi_{g}$.

The CS Algorithm for the optimization of the Log transform parameters is below, where during the algorithm: a) after every iteration, all cuckoos move towards the nest using the Lévy flight approach, b) the number of nests is set after reaching the stopping criteria either a fixed number of iteration or tolerance value), c) the solution is randomly initialized by generating $w$ different nests (solutions), d) fitness for each of the obtained solutions is evaluated. Find the best nest corresponding to minimum/maximum (according to minimization or maximization) value of fitness. The fitness of the solution should be linked to the objective function of the problem of interest to start iterating and to generate a new nest by Levy flight. A Levy flight can be formed using (15). 


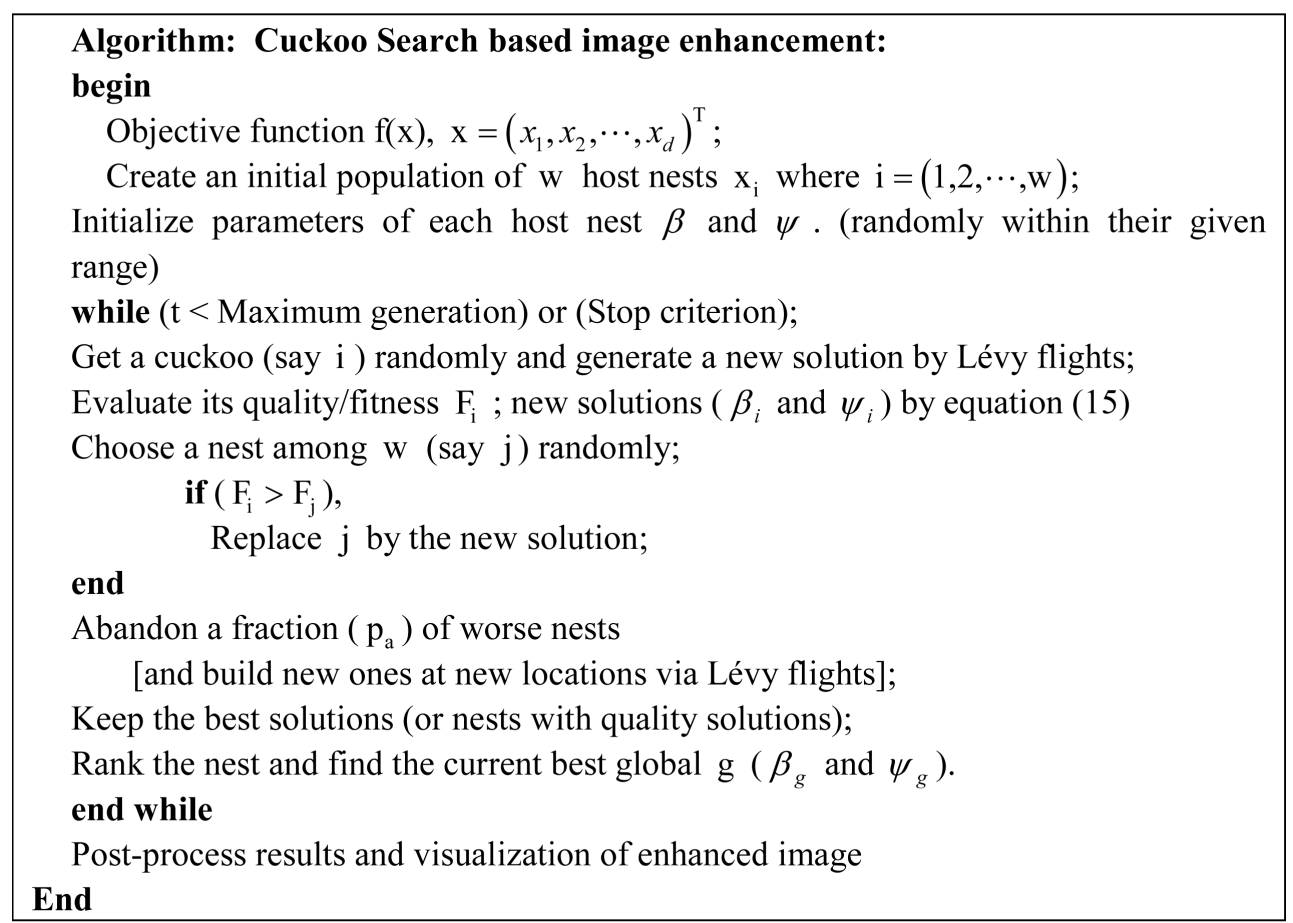

\section{Results and Discussion}

The field of medical image enhancement is a significant aspect of medical image processing. Medical image enhancement techniques are used to improve some useful information in an image for physicians' accurate diagnoses and removal/reduction of some unwanted information. Recently, numerous studies are performed in the medical imaging domain as in [51]-[54].

Therefore, the proposed work is to study the Computed Tomography image enhancement using extensively the MATLAB 2012a software. The medical image enhancement of the proposed system uses log transform based cuckoo search algorithm and gray level images. The algorithm has been executed at a base station in a Windows 7 platform having 4GB RAM, Intel Core i7 $2.80 \mathrm{GHz}$ processor. The proposed method is tested on a database of 51 abdominal, low contrast/quality CT gray level liver lesions images taken from the Scientific Research Group in Egypt database (http://egyptscience.net/).

\subsection{Comparing the results of Cuckoo Search versus PSO Algorithm}

Generally, there some limitations regarding the computational time consuming that the metaheuristic algorithms suffer from, where the parameter tuning is considered a time-consuming process for tuning algorithms. As the wide range of problems try to find the best tune of the metaheuristic algorithm [55].

A comparison between CT of CS and CT of PSO is shown in Figure 1 to illustrate the results obtained from CT multiple liver lesions.

Another comparison set for the CT multiple liver lesions is shown in Figure 2. This figure shows a different assessment when using the CS or the PSO compared with the original CT images.

Figure 1 and Figure 2 established that the using CS provides more enhanced images than that are obtained using PSO. It is clear that the enhanced images using CS for the log transformation parameters optimization are clearer and include all portions of the liver with obvious lesion appearance compared to those based on PSO.

The comparison is done to benchmark the proposed cuckoo search algorithm with the particle swarm optimization based approach. Table 1 shows a comparison between the fitness of the PSO and that of the CS to optimize the two parameters $\beta$ and $\psi$. It is performed up to 20 iterations and reports the number of iterations versus PSO and CS for the two parameter values as well as the fitness. This table illustrates that the fitness of the CS is better than that obtained using PSO for each corresponding iteration where,

1) The fitness value using the CS converges from the 13th iteration. Upon further iterations, the CS has pro- 


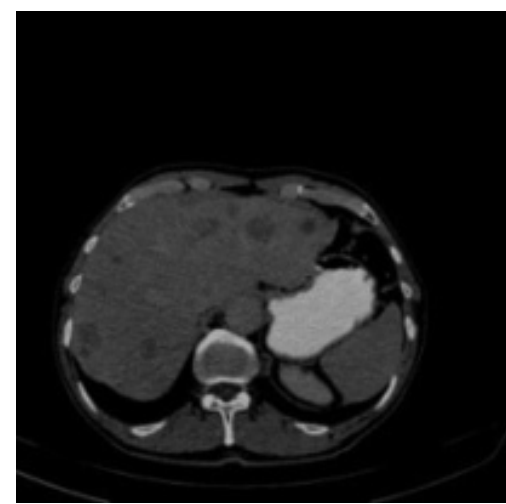

(a)

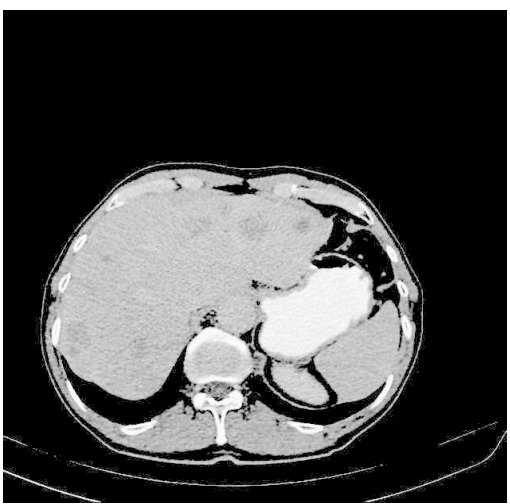

(b)

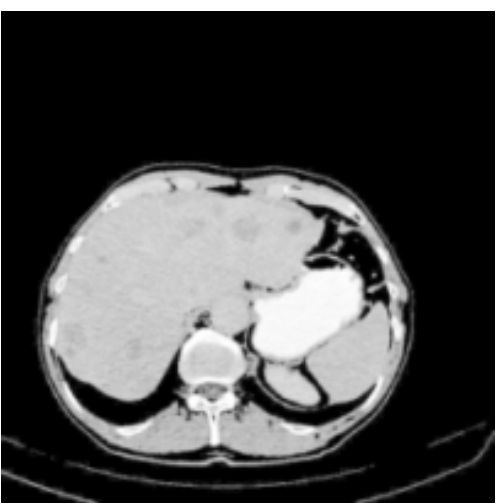

(c)

Figure 1. Output images using PSO and CS compared to the original image: (a) the original image; (b) the enhanced image based on PSO algorithm and (c) the enhanced image based on the Cuckoo Search algorithm.

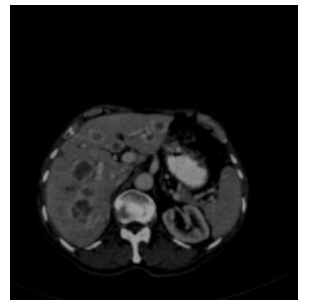

(a)

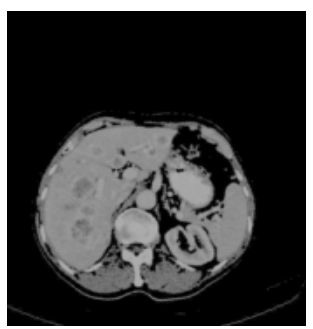

(f)

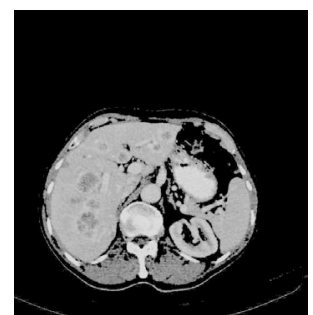

(k)

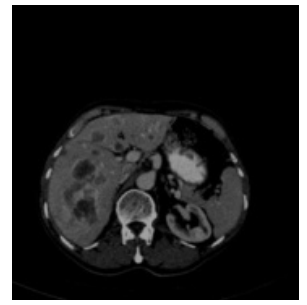

(b)

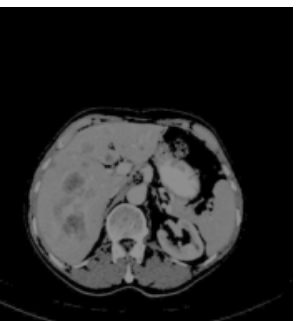

(g)

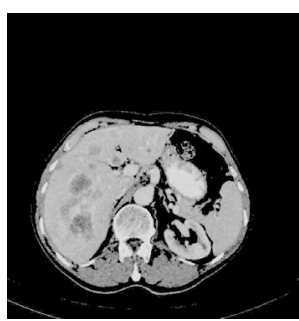

(l)

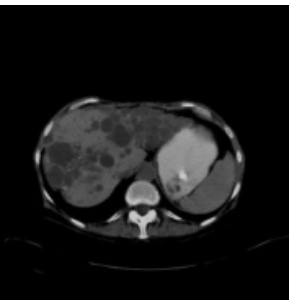

(c)

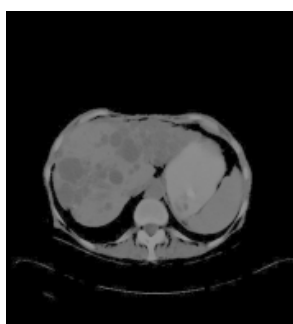

(h)

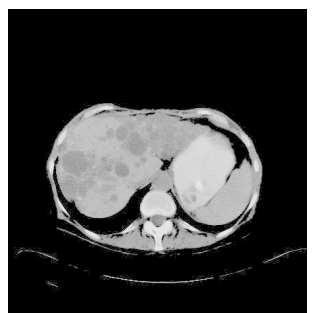

(m)

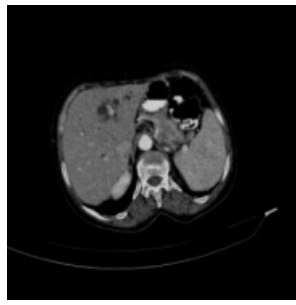

(d)

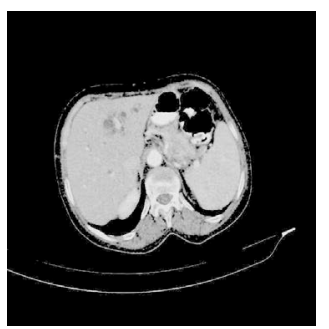

(i)

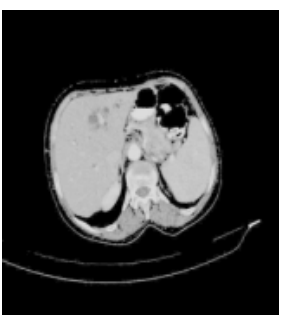

(n)

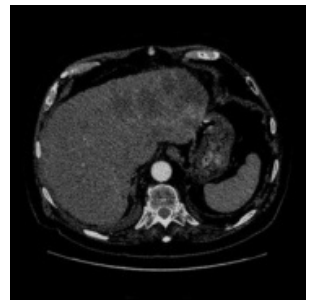

(e)

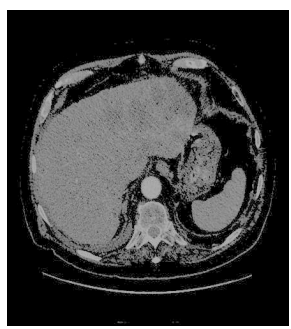

(j)

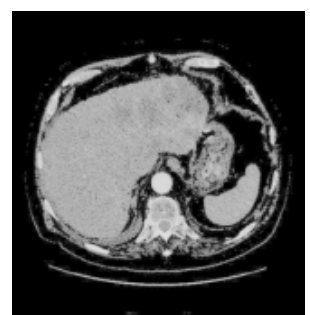

(o)

Figure 2. (a)-(e) Original image; (f)-(j) enhanced based PSO; (k)-(o) enhanced based Cuckoo search.

duced the same fitness values.

2) The PSO doesn't converge up to the 20th iteration. Also, the fitness value of the 20th iteration is less than that obtained using CS.

Table 1 and Figure 3, clearly reports that CS is superior to PSO in terms of both better fitness value and less time consuming to converge by studying the parameters namely: $\beta$ and $\psi$.

\subsection{Image Quality Analysis}

According to the National Imagery Interpretability Rating Scale (NIIRS) [56], image quality can be viewed as a 


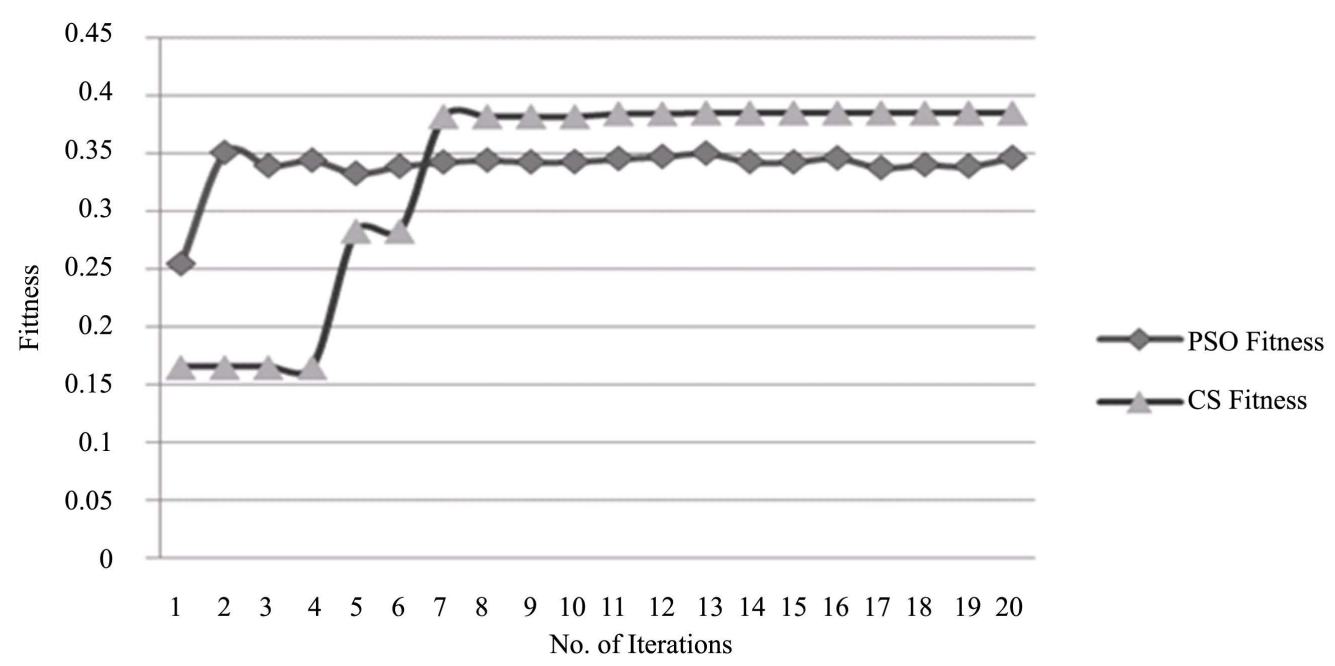

Figure 3. The fitness versus the number of iterations.

Table 1. Comparison between PSO and CS.

\begin{tabular}{|c|c|c|c|c|c|c|}
\hline \multirow[b]{2}{*}{ Iteration } & \multicolumn{3}{|c|}{ Particle Swarm Optimization } & \multicolumn{3}{|c|}{ Cuckoo Search } \\
\hline & Value of $\boldsymbol{\beta}$ & Value of $\psi$ & Fitness & Value of $\boldsymbol{\beta}$ & Value of $\psi$ & Fitness \\
\hline 1 & 0.239645 & 0.801125 & 0.254787 & 0.03276 & 0.055063 & 0.164771 \\
\hline 2 & 0.190233 & 0.841175 & 0.350391 & 0.03276 & 0.055063 & 0.164771 \\
\hline 3 & 0.129936 & 0.592165 & 0.339073 & 0.03276 & 0.055063 & 0.164771 \\
\hline 4 & 0.112296 & 0.655119 & 0.343742 & 0.03276 & 0.055063 & 0.164771 \\
\hline 5 & 0.107487 & 0.53455 & 0.332492 & 0.042726 & 1.268582 & 0.282222 \\
\hline 6 & 0.115824 & 0.589853 & 0.338578 & 0.042726 & 1.268582 & 0.282222 \\
\hline 7 & 0.140781 & 0.633538 & 0.34222 & 0.196434 & 1.579214 & 0.380859 \\
\hline 8 & 0.14733 & 0.643457 & 0.343771 & 0.196434 & 1.579214 & 0.380859 \\
\hline 9 & 0.145702 & 0.628194 & 0.342372 & 0.196434 & 1.579214 & 0.380859 \\
\hline 10 & 0.143447 & 0.631918 & 0.342515 & 0.196434 & 1.579214 & 0.380859 \\
\hline 11 & 0.149737 & 0.662162 & 0.345147 & 0.079666 & 1.815622 & 0.383329 \\
\hline 12 & 0.154622 & 0.684663 & 0.346963 & 0.079666 & 1.815622 & 0.383329 \\
\hline 13 & 0.165177 & 0.731784 & 0.349811 & 0.194583 & 1.583505 & 0.384113 \\
\hline 14 & 0.143799 & 0.636281 & 0.342539 & 0.194583 & 1.583505 & 0.384113 \\
\hline 15 & 0.142713 & 0.631166 & 0.342282 & 0.194583 & 1.583505 & 0.384113 \\
\hline 16 & 0.152313 & 0.673288 & 0.34581 & 0.194583 & 1.583505 & 0.384113 \\
\hline 17 & 0.131058 & 0.579379 & 0.337402 & 0.194583 & 1.583505 & 0.384113 \\
\hline 18 & 0.136432 & 0.603201 & 0.339942 & 0.194583 & 1.583505 & 0.384113 \\
\hline 19 & 0.134301 & 0.593873 & 0.338746 & 0.194583 & 1.583505 & 0.384113 \\
\hline 20 & 0.152568 & 0.67466 & 0.346046 & 0.194583 & 1.583505 & 0.384113 \\
\hline
\end{tabular}

task-based scale used for military, civilian, and agricultural applications. It is used to model the metrics between an original image and an enhanced version of that image. The goal of the image quality analysis (IQA) is to evaluate and compare the performance of image processing algorithms. The quality is improved due to the image enhancement. The vast majority of the IQA algorithms is to measure local pixel wise differences and then to 
collapse these local measurements into a scalar which correspond to the overall quality difference [57]. The metrics for IQA are calculated as follows;

1) The Peak Signal to Noise ratio: is the ratio between the signal maximum power and the power of the corrupting noise that influences the consistency of its representation.

$$
P S N R=10 \log _{10}\left(225^{2} / M S E\right)
$$

2) The Maximum Difference: is the difference between any two pixels such that the larger pixel emerges after the smallest pixel. The largest value of maximum difference indicates an image with poor quality.

$$
M D=\operatorname{MAX}\left(\left|x_{j, k}-x_{j, k}^{\prime}\right|\right)
$$

The Structural Content (SC): compares two images in a number of small image patches that the images have in common.

$$
S C=\sum_{j=1}^{M} \sum_{k=1}^{N} x_{j, k}^{2}
$$

In addition, the Mean Square Error is defined as the mean squared diversity between the original image and the deformed image as it is calculated from the Euclidian distance involving the original and the degraded images. Based on the previous IQA metrics, Table 2 compares the image quality of both the CS and the PSO algorithms. Note that for both algorithms, the log transform based image enhancement techniques PSNR, MSE or any IQA factors are not taken into consideration for the computation of the fitness function. It is clearly depicted that CS based technique sometimes gives higher PSNR and less MSE than PSO for the current experimental setup for most cases (because of selection of images, parameter setting, etc.) as shown in Table 2.

It is clear from Table 2, that almost the PSNR gained by the CS algorithm is either better or near the value with the PSO algorithm. Therefore, the motivation of selecting the CS based approach is that it has faster convergence rate and better fitness (Table 1) than that of PSO based approach, often slightly compromising with the image quality.

\section{Conclusions}

Although using Computed Tomography (CT) is very common in medical applications nowadays, it still has some disadvantages. Consequently, in this work, the CT image enhancement system is proposed using a log transformation based cuckoo search optimization algorithm. The results show that using the CS is superior to

\begin{tabular}{lccccc} 
Table 2. Different values of IQA parameters. & $\begin{array}{c}\text { Mean Square } \\
\text { Error }\end{array}$ & $\begin{array}{c}\text { Structural } \\
\text { Content }\end{array}$ & $\begin{array}{c}\text { Maximum } \\
\text { Difference }\end{array}$ & $\begin{array}{c}\text { Peak Signal to } \\
\text { Noise Ratio }\end{array}$ \\
\hline Image No. & & 0.268224 & 1 & $\mathbf{1 6 . 0 4 7 9 2}$ \\
\hline $\begin{array}{l}\text { Figure 1(c) } \\
\text { Figure 1(b) }\end{array}$ & CS & 1615.431 & 0.166428 & 1 & 12.70088 \\
Figure 2(k) & CS & 3491.34 & 0.376256 & 27 & $\mathbf{1 8 . 6 7 5 9 5}$ \\
Figure 2(f) & PSO & 3567.517 & 0.165018 & 2 & 12.60714 \\
Figure 2(l) & CS & 595.5788 & 0.550663 & 68 & $\mathbf{2 0 . 3 8 1 4 1}$ \\
Figure 2(g) & PSO & 2911.217 & 0.228922 & 8 & 13.49006 \\
Figure 2(m) & CS & 2052.392 & 0.306006 & 1 & $\mathbf{1 5 . 0 0 8 2}$ \\
Figure 2(h) & PSO & 2460.211 & 0.277327 & 3 & 14.22108 \\
Figure 2(n) & CS & 4083.985 & 0.203789 & 1 & 12.01996 \\
Figure 2(i) & PSO & 1711.182 & 0.344225 & 75 & 15.79784 \\
Figure 2(o) & CS & 6050.041 & 0.137964 & 1 & 10.31322 \\
Figure 2(j) & PSO & 5664.836 & 0.144281 & 1 & 10.59893 \\
\hline
\end{tabular}


PSO with respect to the convergence rate and the fitness values. As convergence is the outcome of meta-heuristic framework. The CS based approach gives higher fitness than PSO based technique in all iterations ( $\beta$ and $\psi$ ) and the fitness value converges for CS based after the 13th iteration. On the other hand, PSO does not converge even after 20 iterations. All the experimental images are tested to report the robustness of CS based approach in terms of speed giving similar results. Thus, this paper study the effect of mixing different well established methods via using the CS to determine the optimum parameters of the log based enhancement on CT image, is the main objective of the proposed work.

This work can be further extended using multi-objective optimization techniques and can be compared to the obtained CS algorithm based results. Also, the medical image enhancement based CS can be applied to different medical imaging devices such as MRI, X-Ray, etc. Beside CS, there are several other meta-heuristic algorithms, namely: Firefly algorithm (FA), Bat algorithm, etc. which can be applied for the enhancement purpose which is beyond the scope of this study.

\section{References}

[1] Schwering, P., Kemp, R. and Schutte, K. (2013) Image Enhancement Technology Research for Army Applications. Proceedings of SPIE, 8706, (870600-2)-(87060O-11).

[2] Harikrishna, O. and Maheshwari, A. (2012) Satellite Image Resolution Enhancement Using DWT Technique. International Journal of Soft Computing and Engineering (IJSCE), 2, 274-278.

[3] Dirim, M. Aksoy, E. and Özsoy, G. (2009) Remote Sensing and Gis Applications for Monitoring Multi-Temporal Changes of Natural Resources in Bursa-Turkey. Journal of Environmental Sciences, 3, 53-59.

[4] Jiang, H. Lou, B. and Liao, S. (2012) Medical Image Enhancement Method Based on Mode Decomposition. Advances in Multimedia Technology (AMT), 1, 21-31. http://dx.doi.org/10.4156/amt.vol1.issue1.3

[5] Cn, D. and Ma, V. (1988) Information Processing in Medical Imaging. Plenum Press, New York.

[6] Ilango, G. and Gowri, B. (2012) $\varepsilon$-Neighbourhood Median Filters to Remove Speckle Noise from CT-Images. International Journal of Applied Information Systems (IJAIS), 4, 40-46. http://dx.doi.org/10.5120/ijais12-450829

[7] Peli, E. (1990) Contrast in Complex Images. Journal of the Optical Society of America, A7, 2032-2040. http://dx.doi.org/10.1364/JOSAA.7.002032

[8] Saruchi, M. (2012) Comparative Study of Different Image Enhancement Techniques. International Journal of Computers \& Technology, 2, 131-133.

[9] Dey, N., Samanta, S., Chakraborty, S., Das, A., Chaudhuri, S. and Suri, J. (2014) Firefly Algorithm for Optimization of Scaling Factors during Embedding of Manifold Medical Information: An Application in Ophthalmology Imaging. Journal of Medical Imaging and Health Informatics, 4, 384-394. http://dx.doi.org/10.1166/jmihi.2014.1265

[10] Dey, N., Acharjee, S., Biswas, D., Das, A. and Chaudhuri, S. (2012) Medical Information Embedding in Compressed Watermarked Intravascular Ultrasound Video. Transactions on Electronics and Communication, 57.

[11] Dey, N., Chaudhuri, S., Chakraborty, S., Ahmed, S., Dey, G. and Maji, P. (2014) Effect of Trigonometric Functions Based Watermarking on Blood Vessel Extraction: An Application in Ophthalmology Imaging. International Journal of Embedding Systems, In Press.

[12] Araki, T., Ikeda, N., Dey, N., Chakraborty, S., Saba, L., Kumar, D., et al. (2015) A Comparative Approach of Four Different Image Registration Techniques for Quantitative Assessment of Coronary Artery Calcium Lesions Using Intravascular Ultrasound. Computer Methods and Programs in Biomedicine, 118, 158-172. http://dx.doi.org/10.1016/j.cmpb.2014.11.006

[13] Mustafi, A. and Mahanti, P. (2009) An Optimal Algorithm for Contrast Enhancement of Dark Images Using Genetic Algorithm. Computer an Information Science, 208, 1-8.

[14] Agrawal, P., Chourasia, V., Kapoor, R. and Agrawal, S. (2014) A Comprehensive Study of the Image Enhancement Techniques. International Journal of Advance Foundation and Research in Computer (IJAFRC), 1, 85-89.

[15] Maini, R. and Aggarwal, H. (2010) A Comprehensive Review of Image Enhancement Techniques. Journal of Computing, 2, 8-13.

[16] Jain, A. (1991) Fundamentals of Digital Image Processing. Prentice-Hall, Englewood Cliffs.

[17] Mundhada, S. and Shandilya, V.K. (2012) Spatial and Transformation Domain Techniques for Image Enhancement. International Journal of Engineering Science and Innovative Technology (IJESIT), 1, 213-216.

[18] Hossain, F. and Alsharif, M. (2007) Image Enhancement Based on Logarithmic Transform Coefficient and Adaptive Histogram Equalization. Proceedings of the International Conference on Convergence Information Technology, Gyeongju, 
21-23 November 2007, 1439-1444. http://dx.doi.org/10.1109/iccit.2007.258

[19] Jain, R., Kasturi, R. and Schunck, B.G. (1995) Machine Vision. McGraw-Hill International Edition, New York.

[20] Chang, D. and Wu, W. (1998) Image Contrast Enhancement Based on a Histogram Transformation of Local Standard Deviation. IEEE Transactions on Medical Imaging, 4, 518-531. http://dx.doi.org/10.1109/42.730397

[21] Talbi, E. (2009) Metaheuristic: From Design to Implementation. John Wiley \& Sons, Hoboken. http://dx.doi.org/10.1002/9780470496916

[22] Tjahjadi, T. and Celik, T. (2012) Automatic Image Equalization and Contrast Enhancement Using Gaussian Mixture Modelling. IEEE Transactions on Image Processing, 21, 145-156. http://dx.doi.org/10.1109/TIP.2011.2162419

[23] Hole, K., Gulhane, V. and Shellokar, N. (2013) Application of Genetic Algorithm for Image Enhancement and Segmentation. International Journal of Advanced Research in Computer Engineering \& Technology (IJARCET), 2, 13421346.

[24] Voss, S. (2001) Meta-Heuristics: The State of the Art. In: Nareyek, A., Ed., Local Search for Planning and Scheduling, Springer-Verlag, Berlin, 1-23. http://dx.doi.org/10.1007/3-540-45612-0 1

[25] Ballerini, L. (1998) Genetic Snakes for Medical Image Segmentation. Proceedings of the Conference on Mathematical Modeling and Estimation Techniques in Computer Vision, San Diego, 19 July 1998, 284-295. http://dx.doi.org/10.1117/12.323453

[26] Kennedy, J. and Eberhart, R. (1995) Particle Swarm Optimization. Proceedings of the IEEE International Conference on Neural Networks, 4, 1942-1948. http://dx.doi.org/10.1109/ICNN.1995.488968

[27] Gaurav, K. and Bansa, H. (2013) Particle Swarm Optimization (PSO) Technique for Image Enhancement. International Journal of Electronics \& Communication Technology (IJECT), 4, 117-119.

[28] Dey, N., Samanta, S., Yang, X., Das, A. and Chaudhuri, S. (2013) Optimization of Scaling Factors in Electrocardiogram Signal Watermarking Using Cuckoo Search. International Journal of Bio-Inspired Computing, 5, 315-326. http://dx.doi.org/10.1504/IJBIC.2013.057193

[29] Imtiaz, M. and Wahid, K. (2014) A Color Reproduction Method with Image Enhancement for Endoscopic Images. Proceedings of the 2014 Middle East Conference on Biomedical Engineering (MECBME), Doha, 17-20 February 2014, 17-20. http://dx.doi.org/10.1109/mecbme.2014.6783224

[30] Haddad, O., Afshar, A. and Marino, M. (2006) Honey-Bees Mating Optimization (HBMO) Algorithm: A New Heuristic Approach for Water Resources Optimization. Journal of Water Resources Management, 20, 661-680. http://dx.doi.org/10.1007/s11269-005-9001-3

[31] Yang, X. and Deb, S. (2014) Cuckoo Search: Recent Advances and Applications. Neural Computing and Applications, 24, 169-174. http://dx.doi.org/10.1007/s00521-013-1367-1

[32] Wang, S.S., Xia, Y., Liu, Q.G., Luo, J.H., Zhu, Y.M. and Feng, D.D. (2012) Gabor Feature Based Nonlocal Means Filter for Textured Image Denoising. Journal of Visual Communication and Image Representation, 23, 1008-1018. http://dx.doi.org/10.1016/i.jvcir.2012.06.011

[33] Gorai, A. and Ghosh, A. (2009) Gray-Level Image Enhancement by Particle Swarm Optimization. Proceedings of the World Congress on Nature \& Biologically Inspired Computing, Coimbatore, 9-11 December 2009, 72-77.

[34] Gonzalez, R. and Woods, R. (2002) Digital Image Processing. Prentice-Hall, Inc., Upper Saddle River.

[35] Wang, C. (2011) Computer-Assisted Coronary CT Angiography Analysis: From Software Development to Clinical Application. Medical Dissertations, Linköping University, Linköping.

[36] Borsdorf, A., Raupach, R., Flohr, T. and Hornegger, J. (2008) Wavelet Based Noise Reduction in CT-Images Using Correlation Analysis. IEEE Transactions on Medical Imaging, 27, 1685-1703.

[37] Chen, G. and Kegl, B. (2007) Image Denoising with Complex Ridge Lets. Pattern Recognition, 40, 578-585. http://dx.doi.org/10.1016/j.patcog.2006.04.039

[38] Arfia, F., Messaoud, M. and Abid, M. (2010) A New Image Denoising Technique Combining the Empirical Mode Decomposition with a Wavelet Transform Technique. Proceedings of the 17th international Conference on Systems, Signals and Image Processing, Rio de Janeiro, 17-19 June 2010, 514-517.

[39] Ali, S., Vathsal, S. and Lalkishore, K. (2010) An Efficient Denoising Technique for CT Images Using Window-Based Multi Wavelet Transformation and Thresholding. European Journal of Scientific Research, 48, 315-325.

[40] Pooja Rana, P. and Chopra, V. (2015) A Study on Image Enhancement Techniques. International Journal of Advanced Research in Computer and Communication Engineering, 4, 609-611. http://dx.doi.org/10.17148/IJARCCE.2015.45130

[41] Bouaziz, A., Draa, A. and Chikhi, S. (2014) A Cuckoo Search Algorithm for Fingerprint Image Contrast Enhancement. Proceedings of the Second World Conference on Complex Systems (WCCS), Agadir, 10-13 November 2014, 678-685. http://dx.doi.org/10.1109/ICoCS.2014.7060930 
[42] Bhandaria, A.K., Sonia, V., Kumara, A. and Singh, G.K. (2014) Cuckoo Search Algorithm Based Satellite Image Contrast and Brightness Enhancement Using DWT-SVD. ISA Transactions, 53, 1286-1296. http://dx.doi.org/10.1016/j.isatra.2014.04.007

[43] Soham, G., Sourya, R., Utkarsh, K. and Arijit, M. (2014) Gray Level Image Enhancement Using Cuckoo Search Algorithm. Advances in Intelligent Systems and Computing, 264, 275-286. http://dx.doi.org/10.1007/978-3-319-04960-1 25

[44] Babu, R.K. and Sunitha, K.V.N. (2015) Enhancing Digital Images through Cuckoo Search Algorithm in Combination with Morphological Operation. Journal of Computer Science, 11, 7-17. http://dx.doi.org/10.3844/jcssp.2015.7.17

[45] Jourlin, M. and Pinoli, J.C. (1988) A Model for Logarithmic Image Processing. Journal of Microscopy, 149, 21-35. http://dx.doi.org/10.1111/j.1365-2818.1988.tb04559.x

[46] Hassanzadeh, T., Vojodi, H. and Mahmoudi, F. (2011) Non-Linear Grayscale Image Enhancement Based on Firefly Algorithm. Lecture Notes in Computer Science, 7077, 174-181. http://dx.doi.org/10.1007/978-3-642-27242-4_21

[47] Nadezhda, S. and Stephanie, S. (2010) Fast and Efficient Iris Image Enhancement Using Logarithmic Image Processing. Proceedings of the Conference on Biometric Technology for Human Identification VII, Orlando, 5-9 April 2010. http://dx.doi.org/10.1117/12.851351

[48] Kaur, G. and Singh, R. (2014) Sharpening Enhancement of Ultra Sound Images Using Firefly Algorithm. International Journal of Advanced Research in Computer Science and Software Engineering, 4, 1039-1044.

[49] Samanta, S., Dey, N., Das, P., Acharjee, S. and Chaudhuri, S. (2012) Multilevel Threshold Based Gray Scale Image Segmentation Using Cuckoo Search. Proceedings of the International Conference on Emerging Trends in Electrical, Electronics and Communication Technologies-ICECIT, India, 12-23 December 2012, 27-34.

[50] Yang, X. and Deb, S. (2010) Engineering Optimization by Cuckoo Search. International Journal of Mathematical Modeling and Numerical Optimization, 4, 330-343. http://dx.doi.org/10.1504/IJMMNO.2010.035430

[51] Kriti, J.V., Dey, N. and Kumar, V. (2015) Chapter 5: PCA-PNN and PCA-SVM Based CAD Systems for Breast Density Classification. In: Hassanien, A.-E., Fahmy, M. and Grosan, C., Eds., Applications of Intelligent Optimization in Biology and Medicine: Current Trends and Open Problems, Springer, Berlin, 159-177.

[52] Kausar, N., Palaniappan, S., Al Ghamdi, B.S., Samir, B.B., Dey, N. and Abdullah, A. (2015) Systematic Analysis of Applied Data Mining Based Optimization Algorithms in Clinical Attribute Extraction and Classification for Diagnosis of Cardiac Patients. In: Hassanien, A.-E., Grosan, C. and Tolba, M.F., Eds., Applications of Intelligent Optimization in Biology and Medicine, Springer, Dordrecht, 217-231. http://dx.doi.org/10.1007/978-3-319-21212-8_9

[53] Cheriguene, S., Azizi, N., Zemmal, N., Dey, N., Djellali, H. and Farah, N. (2015) Optimized Tumor Breast Cancer Classification Using Combining Random Subspace and Static Classifiers Selection Paradigms. In: Hassanien, A.-E., Grosan, C. and Tolba, M.F., Eds., Applications of Intelligent Optimization in Biology and Medicine, Springer, Dordrecht, 289-304.

[54] Acharjee, S., Dey, N., Samanta, S., Das, D., Roy, R., Chakraborty, S. and Chaudhuri, S.S. (2015) ECG Signal Compression Using Ant Weight Lifting Algorithm for Tele-Monitoring. Journal of Medical Imaging and Health Informatics, 5, 1246-1250.

[55] Yang, X.S., Chien, S.F. and Ting, T.O. (2014) Computational Intelligence and Metaheuristic Algorithms with Applications. Scientific World Journal, 2014, 1-4. http://dx.doi.org/10.1155/2014/425853

[56] Irvine, J.M. (2011) National Imagery Interpretability Rating Scales (NIIRS): Overview and Methodology. SPIE, 3128, 93-103.

[57] Chandler, D.M. (2013) Seven Challenges in Image Quality Assessment: Past, Present, and Future Research. ISRN Signal Processing, 2013, 1-53. http://dx.doi.org/10.1155/2013/905685 